\title{
Article \\ A QR Code-Based Approach to Differentiating the Display of Augmented Reality Content
}

\author{
Pei-Yu Lin ${ }^{1}$, Wen-Chuan $\mathrm{Wu}^{2, *}$ and Jen-Ho Yang ${ }^{3, *}$ \\ 1 Department of Information Communication, Innovation Center for Big Data and Digital Convergence, \\ Yuan Ze University, Taoyuan City 320315, Taiwan; pagelin3@gmail.com \\ 2 Department of Computer Science and Information Engineering, National Ilan University, \\ Yilan County 260007, Taiwan \\ 3 Department of Digital Multimedia Design, Kainan University, Taoyuan City 338103, Taiwan \\ * Correspondence: wenn@niu.edu.tw (W.-C.W.); jenhoyang@mail.knu.edu.tw (J.-H.Y.)
}

check for

updates

Citation: Lin, P.-Y.; Wu, W.-C.; Yang, J.-H. A QR Code-Based Approach to Differentiating the Display of Augmented Reality Content. Appl. Sci. 2021, 11, 11801. https://doi.org/ 10.3390/app112411801

Academic Editor: Jason K. Levy

Received: 29 November 2021 Accepted: 10 December 2021 Published: 12 December 2021

Publisher's Note: MDPI stays neutral with regard to jurisdictional claims in published maps and institutional affiliations.

Copyright: (C) 2021 by the authors. Licensee MDPI, Basel, Switzerland. This article is an open access article distributed under the terms and conditions of the Creative Commons Attribution (CC BY) license (https:// creativecommons.org/licenses/by/ $4.0 /)$.

\begin{abstract}
The augmented reality (AR) system requires markers to recognize and locate virtual objects on the screens of mobile devices. However, both markers and objects must be registered via the online platform in advance. In addition, an AR marker can only pair with a fixed set of virtual objects, limiting the flexibility and immediacy of changing and updating these data. This paper incorporates the quick response barcode (QR code) into the AR system to address these issues. We propose an algorithm with two vital goals, including (1) generating differentiated virtual objects for different target users by using only one QR code as the marker and (2) concealing different private authentication in QR modules by applying the error correction capability. We then demonstrate the proposed approach via a simulation of two practical scenarios, the electronic catalogs for business applications, and differentiated instructional materials for digital learning. This paper contributes to $\mathrm{AR}$ and $\mathrm{QR}$ code research and practices.
\end{abstract}

Keywords: AR; QR code; mobile computing; error correction capability; differentiated display

\section{Introduction}

The rapid advance in the hardware and software of smart devices has benefited the development of augmented reality (AR) technologies. In recent years, AR can operate efficiently without heavy and expensive equipment in different scenarios, such as educational training, digital learning, product marketing, and industrial design [1-3]. Furthermore, by taking AR's capability of associating virtual objects with real space, many studies have applied it to spatial visualization [4]. Examples include combining the localization technique to improve interactive indoor navigation [5], helping people with limited peripheral vision identify things in the environment [6], and enhancing rehabilitation training for older people by providing a visualized guide [7].

In short, AR enables users to see virtual objects and models on their mobile phones. Most AR technologies are marker-based, meaning marker recognition is used to locate the relative position of $3 \mathrm{D}$ virtual models on the screen. Common development tools include ARToolkit, NyARToolKit, and AR Tag [8-10], and markers have different forms, such as frame markers, dot markers, split markers, data Matrix markers, ID markers, and template markers. These markers are usually simple squares, text, or patterns in black and white or grayscale, with the advantage of allowing the system to recognize patterns accurately and quickly when the light is dark or the camera pixels are insufficient.

While AR is convenient, it has several limitations $[4,11-13]$ as follows:

1. The marker and the virtual object must be registered and linked in advance on the online platform to track the relative position and direction between the camera and the tag, download the linked virtual object, and display it. In this case, the marker 
and the object cannot change dynamically, significantly limiting the effectiveness and immediacy.

2. Keeping the internet connection is necessary to access the AR system and download virtual objects in real-time.

3. The content, card size, image feature points, or print size of markers must meet certain conditions to maintain the system's stability.

4. Different companies have marker patterns that are not interchangeable, making it impossible to share sources and enable compatibility.

As shown in recent studies $[5,14]$, the trend of AR would provide real-time computed and flexible media and content to let users interact with various virtual objects. Therefore, it is vital to resolve the limitations of the marker-based AR technique.

Combining AR with the quick response barcode ( $Q R$ code) can be a gateway to address the above issues. The $\mathrm{QR}$ code is a two-dimensional matrix developed by Denso-Wave company in $1994[15,16]$. Based on this technique, the data content of a QR tag can be encoded and decoded efficiently. Therefore, more and more research has tried to combine mobile AR with QR codes [17,18]. For example, Ken et al. [17] applied QR code as an AR-based marker to track the angle of the QR Code and its positioning coordinates and mapped 3D virtual objects to the QR marker. VisionLens [18] also utilized a similar concept, establishing a QR Code for each product. After scanning the QR code, the AR virtual object and $3 \mathrm{D}$ animation will appear when mobile devices point at the product. In brief, the data content of the QR code can be a link to a virtual object or a uniform resource locator (URL). Therefore, when triggering the AR system, the location information of the QR marker can be estimated immediately and decode the data content of the URL to retrieve 3D virtual objects. Moreover, this process does not require users' pre-registration of the AR system.

Unfortunately, current AR schemes [17-22] align the same virtual object with the same marker. The AR system thus considers all users with the same maker the same role. In other words, the AR system can only display the same virtual content to various users. However, companies often need to deliver different information to different target customers in real-world business applications. Accordingly, we propose a new AR mechanism to present differentiated virtual objects for target users by using the same QR marker to deal with this issue [23]. This approach is practical and can meet different scenarios. For example, companies can display an ordinary virtual object to general customers and deliver advanced models such as recommended products or preferences to the jointed member or VIP. In addition, the proposed approach can also provide differentiated instruction for educational purposes, such as offering students of different levels different learning materials. Therefore, this paper develops a flexible and differentiated AR system.

\section{Design of Differentiated AR System Based on QR Markers}

Figure 1 presents a conceptual model of our approach. To show the differentiated $3 \mathrm{D}$ virtual objects in the AR system, we develop a secret $Q R$ tag generation procedure in Section 2.1. Then we describe how the $Q R$ tag enables AR recognition and the presentation of specific virtual objects in Section 2.2.

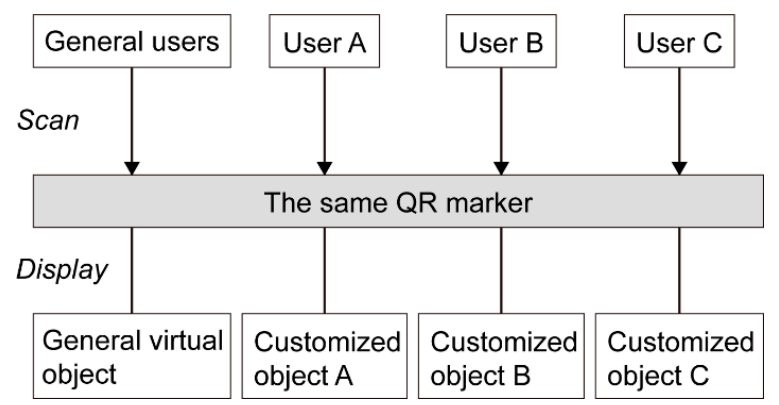

Figure 1. A conceptual model of the proposed differentiated AR system. 


\subsection{Secret $Q R$ Tag Generation}

We designed a secret $\mathrm{QR}$ tag concealment algorithm to derive the marked $\mathrm{QR}$ tag by applying the concept of the steganography technique [23-25]. Let $Q$ be a given $Q R$ tag generated by a barcode generator. Here, the data content of $Q$ can be a link or a uniform resource locator (URL) of the general virtual object. Public users can receive the ordinary virtual object from the QR tag.

According to the QR barcode standard, let $n$ be the number of QR data and error correction modules, as the red region in Figure 2. To distinguish the general user and the specific users, let there are $m$ classifications of members, $I D_{i}$ and $s_{i}$ be the identification number and the authentication code of the $i$-th member classification, $1 \leq i \leq m$. Here, the value of $s_{i} \in\{0,1\}$ and the length of $s_{i}$ is $\left\lceil\log _{2} m\right\rceil$.

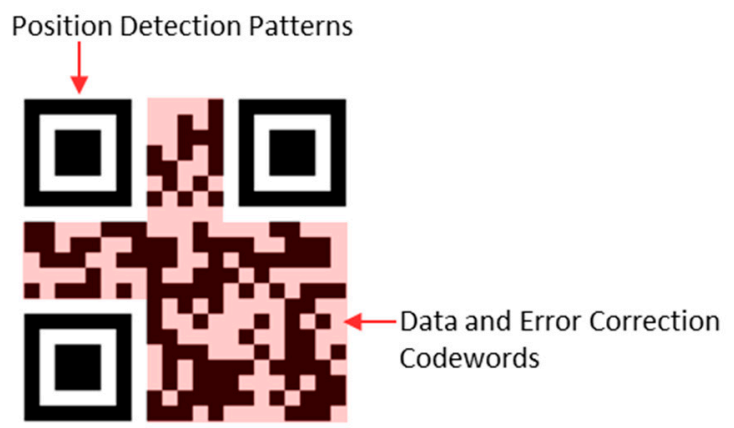

Figure 2. The modules of data and error correction codewords of QR tag.

The stream $c_{i}$ corresponding to $I D_{i}$ thereby can be generated by

$$
c_{i}=H_{K}\left(I D_{i}\right) \bmod n .
$$

Here, $K$ is the secret key of the system/dealer, and $H_{K}\left({ }^{\bullet}\right)$ is the one-way hash function with the key $K$. The stream length of $c_{i}$ is $\left\lceil\log _{2} m\right\rceil$. The $c_{i}$ indicates the concealed positions of the QR modules for the $i$-th member. Note that the generated values of $c_{i}$ are limited within $n$, and the values in $c_{i}$ cannot be selected more than once to avoid overlapping.

Assume $q_{i}$ are the selected modules in $Q$ according to the assigned positions $c_{i}$. The authentication codes $s_{i}$ for differential members thereby are concealed into the corresponding $q_{i}$ modules by

$$
q_{i}^{\prime}=q_{i} \oplus s_{i},
$$

where $q_{i}{ }^{\prime}$ are labeled as the modified modules. Note that, to conceal $s_{i}$ into the QR tag without degrading the readability of the content, the value of $m$ should be restricted,

$$
m \times\left\lceil\log _{2} m\right\rceil \leq\lfloor E C / 2\rfloor \times 8 .
$$

Here, $E C$ be the number of error correction codewords of the given $Q$.

With the concealment process, the embedded $Q R \operatorname{tag} Q^{\prime}$ consequently can be derived. Figure 3 demonstrates the result of the marked QR tag generation procedure for eight classifications of members (i.e., $m=8$ ). The original $Q R$ tag and the marked $Q R$ tag are depicted in Figure 3a,b, respectively. The red modules in Figure 3c show the difference between Figure $3 a, b$. 


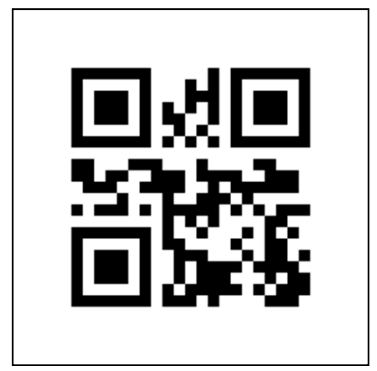

(a)

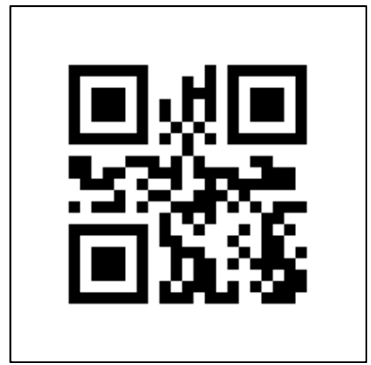

(b)

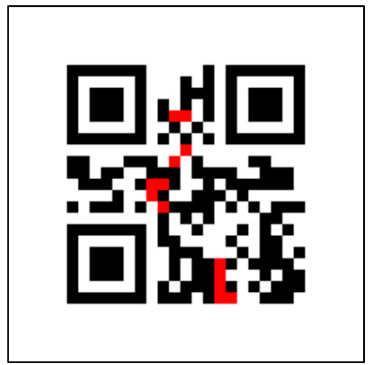

(c)

Figure 3. The results of the marked QR tag. (a) The original QR tag. (b) The marked QR tag. (c) The difference between (a) and (b).

\subsection{QR Tag-Based AR Procedure}

To recognize and locate the virtual objects upon the marked $Q R \operatorname{tag} Q^{\prime}[17,24]$, the position detection patterns of the QR tag (the black and white pattern in Figure 4a) is firstly registered as the marker in ARToolKit [13]. The camera capture can detect the three-position detection patterns $A_{0}, B_{0}$, and $C_{0}$, as depicted in Figure $4 \mathrm{~b}$. To find the fourth corner, we apply the corner-based manner to find $D_{0}$ according to $A_{0}, B_{0}$, and $C_{0}$. Figure $4 \mathrm{c}$ shows the estimated fourth corner [24].

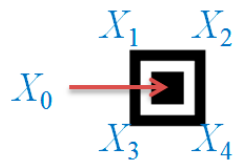

(a)

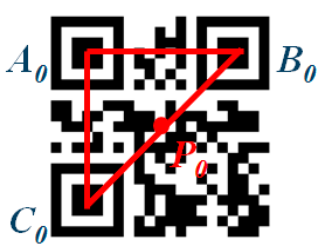

(b)

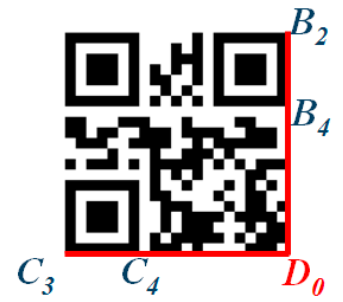

(c)

Figure 4. The corners finding process of a QR tag [24]. (a) The position detection patterns of the QR tag. (b) The three-position detection patterns $A_{0}, B_{0}$, and $C_{0}$. (c) The derived fourth corner $D_{0}$.

With the four corners, the camera pose can be estimated by exploiting homography between the captured image and the established feature map of $Q^{\prime}$. Finally, we can estimate the rotation degree $R$ and the translation $T$ between camera and $Q^{\prime}$.

With the marked $Q^{\prime}$, the camera pose, such as the rotation matrix $R$ and the translation $T$, can be calculated. According to the $Q R$ content of $Q^{\prime}$, the normal 3D virtual object can be rendered on the scene by transforming the $3 \mathrm{D}$ virtual object with $R$ and $T$. Moreover, the specific member with their member number $I D_{i}$ can further extract the corresponding authentication code by the system key $K$ automatically. The system thereby can link and render the specific $3 \mathrm{D}$ virtual objects for differential members.

\section{Demonstration of the Proposed Approach}

To demonstrate the proposed method, we evaluate the reliability by scanning the marked QR tag in different lighting conditions with different devices and software in Section 3.1 and show the usability by turning our algorithm into practical systems for meeting different scenarios in Section 3.2.

\subsection{Reliability Evaluation}

Different mobile devices, scanning software, and environmental illumination may influence the accuracy of QR content decoding. Hence, we assess the feasibility of our system via three different smartphones (two in the Android system and one in the iOS system) and four types of scanning software under three different lighting conditions. 
In the scanning tests, we evaluate QR codes displayed both on the screen and the printed paper using two QR tags generated by our method (see Figure 5). Table 1 shows the scanning results via two phones of the Android system (HTC: Desire 20 Pro and Samsung: Note 20), while Table 2 shows the results of the iOS system (iPhone 11 Pro MAX). We set three lighting conditions: the outdoor, the indoor with living room lights, and the indoor without artificial lights. The illuminance values captured in this test are lux $=305,221$, and 21 , respectively. As shown, both two QR codes can be decoded by four types of scanning software under three sets of environmental illumination.

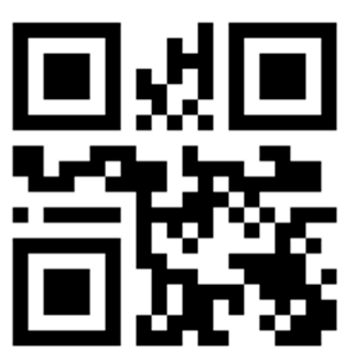

(a)

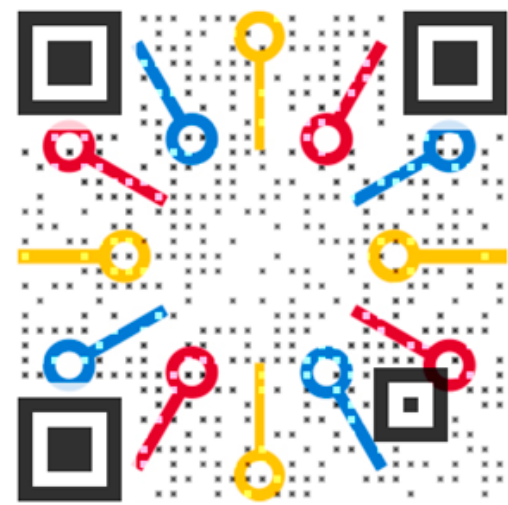

(b)

Figure 5. Two QR tags generated by our method for the scanning test: (a) QR data content: Applied Sciences. QR version: 1, Error correction level: L, authentication length: 24 bits; (b) QR data content [26]: http:/ / www.infocom.yzu.edu.tw/ (accessed on 10 December 2021). QR version: 4, Error correction level: $\mathrm{H}$, authentication length: 256 bits.

Table 1. Scanning tests of the Android system under different lighting conditions.

\begin{tabular}{lcccccc}
\hline Mobile Device & \multicolumn{3}{c}{ HTC } & \multicolumn{3}{c}{ Samsung } \\
\hline \multirow{2}{*}{ Light Intensity (lux) } & Outdoor & \multicolumn{2}{c}{ Indoor } & Outdoor & Indoor \\
\cline { 2 - 8 } & 305 & 221 & 21 & 305 & 221 & 21 \\
\hline \multirow{2}{*}{ Media Type of QR Code } & $\begin{array}{l}\text { Print/ } \\
\text { Screen }\end{array}$ & Print/ & Print/ & Print/ & Print/ & Print/ \\
\hline QR Code \& Barcode Scanner & $\checkmark$ & $\checkmark$ & $\checkmark$ & $\checkmark$ & $\checkmark$ & $\checkmark$ \\
\hline QR Code Reader & $\checkmark$ & $\checkmark$ & $\checkmark$ & $\checkmark$ & $\checkmark$ & $\checkmark$ \\
\hline QR Code Reader-Scanner App & $\checkmark$ & $\checkmark$ & $\checkmark$ & $\checkmark$ & $\checkmark$ & $\checkmark$ \\
\hline Scandit Barcode Scanner Demo & $\checkmark$ & $\checkmark$ & $\checkmark$ & $\checkmark$ & $\checkmark$ & $\checkmark$ \\
\hline
\end{tabular}

Table 2. Scanning tests of the iOS system under different lighting conditions.

\begin{tabular}{lccc}
\hline \multirow{2}{*}{ Light Intensity (lux) } & Outdoor & \multicolumn{2}{c}{ Indoor } \\
\cline { 2 - 4 } Media Type of QR Code & $\mathbf{3 0 5}$ & $\mathbf{2 2 1}$ & $\mathbf{2 1}$ \\
\hline QR Code \& Barcode Scanner & Print/Screen & Print/Screen & Print/Screen \\
\hline QR Code & $\checkmark$ & $\checkmark$ & $\checkmark$ \\
\hline QR Square & $\checkmark$ & $\checkmark$ & $\checkmark$ \\
\hline Scandit Barcode Scanner Demo & $\checkmark$ & $\checkmark$ & $\checkmark$ \\
\hline
\end{tabular}




\subsection{Simulation Results}

We conducted simulations to demonstrate the usability of our system. Figures 6 and 7 are our simulation results in different scenarios. This simulation uses Vuforia Developer [27] to build the AR content with the processed QR code (Figure 5b). After installing the designed AR app, users can obtain these 3D objects by scanning the QR code.

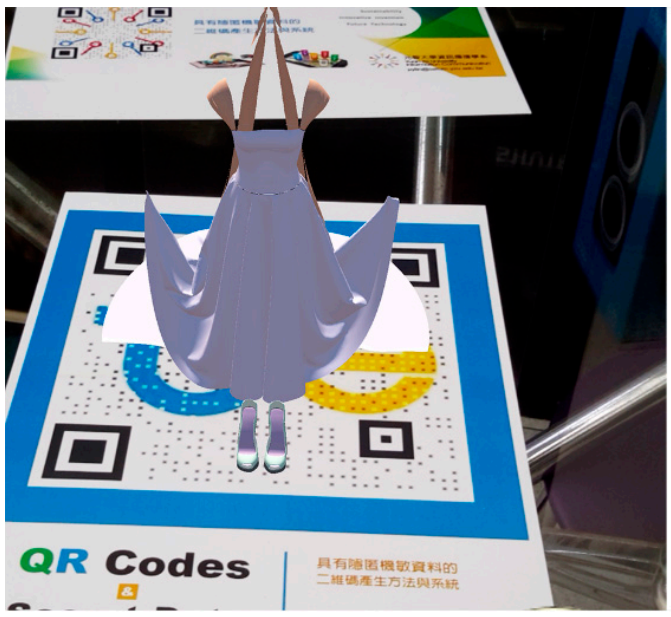

General customers with standard virtual object

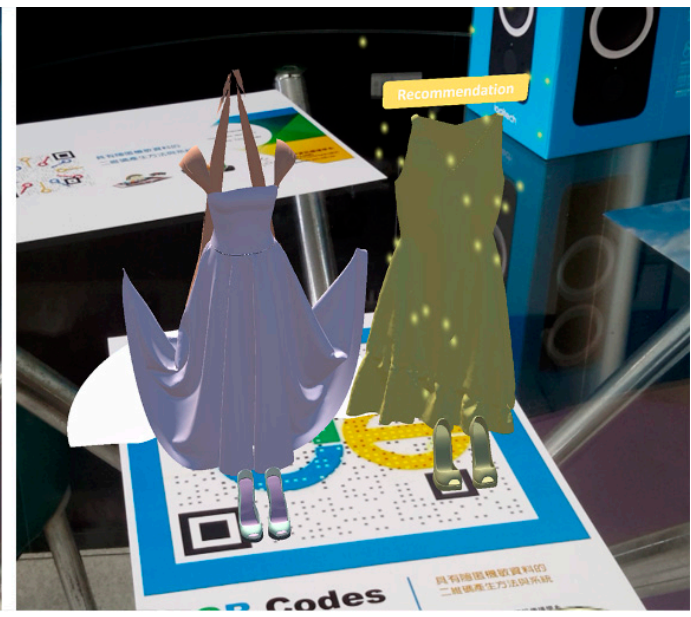

Specific customers with the customized object

Figure 6. The simulation results of the proposed approach in the scenario of fashion design store.

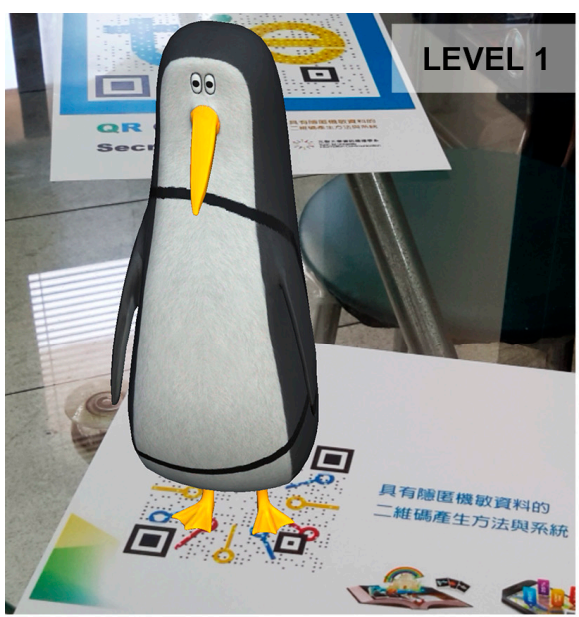

Low-level learners with simple material

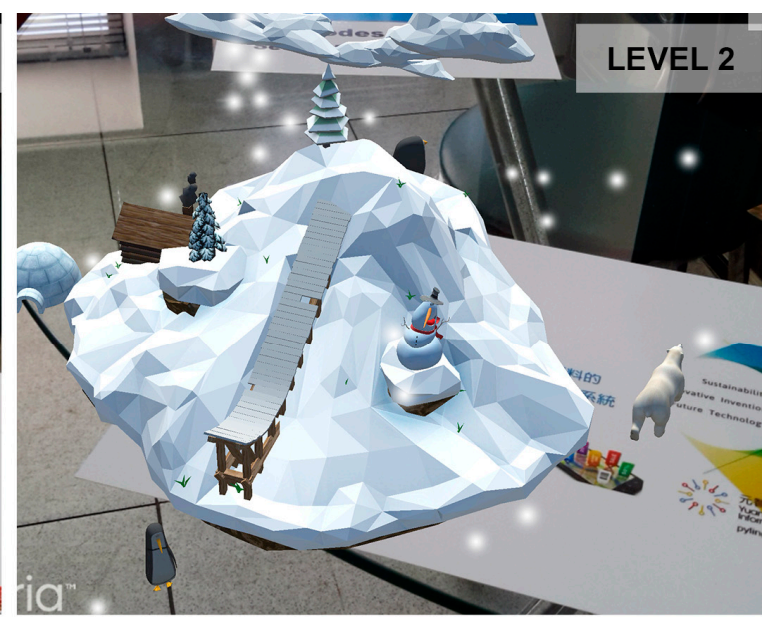

Advanced learners with complex materials

Figure 7. The simulation results of the proposed approach in the scenario of digital learning.

Figure 6 presents a fashion design store that provides differentiated electronic catalogs based on clients' attributes via the same QR code. The left side of Figure 6 shows the augmented reality results that general users see when scanning the $Q R$ code. The right side of Figure 6 offers a recommended product in addition to the standard one for specific users.

In our system, when a user installs the app, he/she will get an identification (i.e., ID indicated in Section 2.1) and an authentication code (i.e., $s_{i}$ ) in his/her mobile device. According to the $I D$, the system $K$ can automatically calculate where the $s_{i}$ information should hide in the QR code. That is, in Figure 6, general users' and specific users' authentication information have been hidden in different positions of the QR code, respectively. The app will trigger corresponding 3D objects only when the ID matches the authentication code.

On the other hand, Figure 7 shows electronic materials for digital learning. These materials are for language learning for children. The left side of Figure 7 has only one simple model, which lets beginners recognize it quickly. Comparatively, the right side 
presents a complicated set of models, requiring advanced learners to identify each of them. Accordingly, with one QR code, different users can receive what they need, thus saving costs and easing the management of numerous images.

\section{Discussions}

The proposed system has combined confidential QR codes and AR. Hence, when users scan the marker, they are no longer limited in getting the same and fixed virtual objects. Besides, the system can present various AR effects according to different user groups or preferences.

Past studies [11-13] focus on improving the recognition of AR without using markers. However, these approaches still require pre-registration to match the image features and virtual objects. Furthermore, these 3D objects remain fixed and cannot update promptly after the registration. The stability and accuracy of scanning are also lower than markerbased techniques. That is why we combine the QR code-based approach with the AR system to break these limitations.

Several studies [17-19] have explored similar issues. However, their emphasis is on recognizing $Q R$ markers, especially positioning the $A R$ on $Q R$ codes after calculating the coordinates. We build our theory upon these studies, but we do not just treat QR codes as markers. Instead, we further incorporate the authentication mechanism to propose the differentiated display of AR content, which is the significant contribution of this study.

Finally, research $[21,22]$ uses multi-markers to provide different AR content in different environments. Compared to their results, our system only requires one $Q R$ code to generate various virtual objects, thus saving costs on producing extra QR markers.

\section{Conclusions and Future Research}

This applied research is practical and could be widely used in many cases. For example, general users can scan the original $Q R$ content data to download AR virtual objects. Companies can update these objects anytime. Specific groups or members can also retrieve different authentication codes to generate specific virtual objects. In addition, the proposed algorithm complies with the QR Code compilation and decoding specifications. Thus, users can scan it with general barcode devices without additional assistance from the computer image processing. In addition to maintaining the original readability, the proposed approach might also benefit confidentiality, security, and visual design of QR code information since it can hide data in an ordinary image. We expect this technical research can contribute to AR research and practical business or education applications.

There are several issues researchers can explore in the future. For example, since our approach provides differentiated AR content to different user groups, it requires more capacity to embed more authentication codes when user groups increase. However, higher $\mathrm{QR}$ code versions and more giant $\mathrm{QR}$ markers are usually needed to hide more information. Thus, it is worth studying how to increase the capacity of the authentication length within the general-sized QR codes. On the other hand, researchers could apply the technique of embedding authentication codes into general images in place of $Q R$ codes, providing another medium for differentiated displays.

Author Contributions: Conceptualization, P.-Y.L. and W.-C.W.; methodology, P.-Y.L. and W.-C.W.; software, P.-Y.L.; validation, J.-H.Y.; formal analysis, J.-H.Y.; resources, P.-Y.L.; writing—original draft preparation, P.-Y.L. and W.-C.W.; writing-review and editing, P.-Y.L. and W.-C.W.; visualization, J.-H.Y.; supervision, J.-H.Y.; project administration, P.-Y.L. All authors have read and agreed to the published version of the manuscript.

Funding: This research was funded by the Ministry of Science and Technology, Taiwan, under Grant MOST 109-2221-E-155-032 and MOST 110-2221-E-155-026-MY3.

Institutional Review Board Statement: Not applicable.

Informed Consent Statement: Not applicable. 
Acknowledgments: We want to thank the anonymous reviewers and Chin-Hung Teng for their valuable comments that helped improve this paper. We also thank Editors Jason K. Levy and Kiki Wang for their efficient work and help. Finally, we are deeply grateful to Chong-Wen Chen for his full and in-time support and constructive suggestions that help complete this paper.

Conflicts of Interest: The authors declare no conflict of interest.

\section{References}

1. Cardoso, L.F.D.; Mariano, F.C.M.Q.; Zorzal, E.R. A survey of industrial augmented reality. Comput. Ind. Eng. 2020, 139, 106159. [CrossRef]

2. Siriwardhana, Y.; Porambage, P.; Liyanage, M.; Ylianttila, M. A Survey on Mobile Augmented Reality With 5G Mobile Edge Computing: Architectures, Applications, and Technical Aspects. IEEE Commun. Surv. Tutor. 2021, 23, 1160-1192. [CrossRef]

3. Jang, J.; Ko, Y.; Shin, W.S.; Han, I. Augmented reality and virtual reality for learning: An examination using an extended technology acceptance model. IEEE Access 2021, 9, 6798-6809. [CrossRef]

4. Dickmann, F.; Keil, J.; Dickmann, P.L.; Edler, D. The impact of augmented reality techniques on cartographic visualization. KN-J. Cartogr. Geogr. Inf. 2021, 1-11. [CrossRef]

5. Ma, W.; Zhang, S.; Huang, J. Mobile augmented reality based indoor map for improving geo-visualization. PeerJ Comput. Sci. 2021, 7, e704. [CrossRef] [PubMed]

6. Angelopoulos, A.N.; Ameri, H.; Mitra, D.; Humayun, M. Enhanced depth navigation through augmented reality depth mapping in patients with low vision. Sci. Rep. 2019, 9, 1-10. [CrossRef]

7. Hoe, Z.Y.; Chen, C.H.; Chang, K.P. Using an augmented reality-based training system to promote spatial visualization ability for the elderly. Univ. Access Inf. Soc. 2019, 18, 327-342. [CrossRef]

8. ARToolKit. Available online: http://www.hitl.washington.edu/artoolkit/ (accessed on 10 December 2021).

9. Nyatla. NyARToolkit Project. Available online: http:/ / nyatla.jp/nyartoolkit/wp/?page_id=198 (accessed on 10 December 2021).

10. ARTage. Available online: https:/ / en.wikipedia.org/wiki/ARTag (accessed on 10 December 2021).

11. Gorovyi, I.M.; Sharapov, D.S. Advanced image tracking approach for augmented reality applications. In Proceedings of the 2017 Signal Processing Symposium (SPSympo), Jachranka, Poland, 12-14 September 2017.

12. Maidi, M.; Lehiani, Y.; Preda, M. Open augmented reality system for mobile markerless tracking. In Proceedings of the IEEE International Conference on Image Processing (ICIP), Abu Dhabi, United Arab Emirates, 25-28 October 2020; pp. $2591-2595$.

13. Cheng, Q.; Zhang, S.; Bo, S.; Chen, D.; Zhang, H. Augmented reality dynamic image recognition technology based on deep learning algorithm. IEEE Access 2020, 8, 137370-137384. [CrossRef]

14. Qian, W.; Coutinho, R.W. On the design of edge-assisted mobile IoT augmented and mixed reality applications. In Proceedings of the 17th ACM Symposium on QoS and Security for Wireless and Mobile Networks, Alicante, Spain, 22-26 November 2021; pp. 131-136. [CrossRef]

15. Denso Wave. Available online: https://www.qrcode.com/en/index.html (accessed on 10 December 2021).

16. ISO/IEC 18004. Information Technology Automatic Identification and Data Capture Techniques Bar Code Symbology QR Code; International Organization for Standardization (ISO): Geneva, Switzerland, 2000.

17. Kan, T.W.; Teng, C.H.; Chou, W.S. Applying QR Code in augmented reality applications. In Proceedings of the International Conference on Virtual Reality Continuum and Its Applications in Industry, Brisbane, Australia, 14-16 November 2019 ; pp. $253-257$.

18. VisionLens. Available online: https:/ / apkpure.com/visionlens/tw.thinkwing.visionlens (accessed on 10 December 2021).

19. Li, H.; Si, Z. Application of augmented reality in product package with quick response code. In Advances in Graphic Communication, Printing and Packaging; Springer: Singapore, 2019; pp. 335-342.

20. Zhang, L.; Sun, A.; Shea, R.; Liu, J.; Zhang, M. Rendering multi-party mobile augmented reality from edge. In Proceedings of the 29th ACM Workshop on Network and Operating Systems Support for Digital Audio and Video, Amherst, MA, USA, 21 June 2019; pp. 67-72.

21. Wada, T.; Kishimoto, Y.; Nakazawa, E.; Imai, T.; Suzuki, T.; Arai, K.; Kobayashi, T. Multimodal user interface for QR code based indoor navigation system. In Proceedings of the IEEE 9th Global Conference on Consumer Electronics (GCCE), Kobe, Japan, 13-16 October 2020; pp. 343-344.

22. Sing, A.L.L.; Ibrahim, A.A.A.; Weng, N.G.; Hamzah, M.; Yung, W.C. Design and development of multimedia and multi-marker detection techniques in interactive augmented reality colouring book. Comput. Sci. Technol. 2020, 603, 605-616.

23. Lin, P.Y.; Teng, C.H.; Chen, Y.H. Diverse augmented reality exhibitions for differential users based upon private quick response code. In Proceedings of the 2015 Asia-Pacific Signal and Information Processing Association Annual Summit and Conference (APSIPA), Hong Kong, China, 16-19 December 2015; pp. 1121-1125. [CrossRef]

24. Lin, P.Y.; Teng, C.H. Differential Augmented Reality System Based on Secret Quick Response Barcode. In Proceedings of the 28th IPPR Conference on Computer Vision, Graphics, and Image Processing (CVGIP 2015), Yilan, Taiwan, 17-19 August 2015.

25. Chiang, Y.J.; Lin, P.Y.; Wang, R.Z.; Chen, Y.H. Blind QR Code Steganographic approach based upon error correction capability. KSII Trans. Internet Inf. Syst. 2013, 7, 2527-2543.

26. Department of Information Communication, Yuan Ze University. Available online: http:/ /www.infocom.yzu.edu.tw/ (accessed on 10 December 2021).

27. Vuforia Engine. Available online: https://developer.vuforia.com/ (accessed on 10 December 2021). 